\title{
LA ECONOMÍA MORAL DEL CUERPO EN EL ANTIGUO RÉGIMEN HISPANO-COLONIAL
}

\author{
THE MORAL ECONOMY OF THE BODY IN \\ THE OLD HISPANIC-COLONIAL REGIME
}

\author{
Grit Kirstin Koeltzsch ${ }^{1}$ (iD \\ Universidad Nacional de Jujuy (Argentina)
}

DOI: https://doi.org/10.17533/udea.tempus.n10a03

Cómo citar este artículo en Chicago Manual of Style: Koeltzsch, Grit Kirstin. "La economía moral del cuerpo en el Antiguo Régimen hispano-colonial". Tempus Revista en Historia General no. 10 (2019):1-23.

Artículo recibido el 1 de septiembre y aprobado para su publicación el 19 de octubre de 2019.

\footnotetext{
${ }^{1}$ UE-CISOR/CONICET, Universidad Nacional de Jujuy. Becaria doctoral de CONICET, Magister en Teoría y Metodología de las Ciencias Sociales, Licenciada en Antropología. Correo electrónico: kirstinkoeltzsch@gmail.com.
} 


\title{
Resumen
}

El concepto de economía moral ha adquirido diversos significados y así también penetrado los estudios sociales de diversas disciplinas. Este trabajo intenta explorar su aplicación antropológica relacionada a las expresiones corporales, en concreto se analizan algunos aspectos sobre el control corporal en el distrito de Jujuy en el siglo XVIII, basándose en fuentes documentales como autos, bandos de Buen Gobierno, ordenanzas y representaciones. Estos reglamentos tienen la particularidad de ser reproducidos o bien producidos localmente por los cabildos de las ciudades, por lo que nos informan de la realidad local del cabildo y el vecindario en consideración a la realidad universal de la Corona. Se propone examinar diversas dimensiones sociales relacionadas a los cuerpos y así revisar el uso del modelo de Thompson y Scott y su aplicación para relacionar prácticas y expresiones corporales con las reglamentaciones de las autoridades coloniales del siglo XVIII. De esta manera la noción de economía moral puede ser utilizada en un sentido más amplio para observaciones analíticas con relación al cuerpo del ser humano.

Palabras clave: Economía moral; cuerpo; control; Jujuy; Antiguo Régimen.

\begin{abstract}
The concept of moral economy has acquired diverse meanings and thus penetrated the social studies of different disciplines. This article attempts to explore its anthropological application related to corporal expressions analyzing some aspects of bodily control in the district of Jujuy in the 18th century. The research is based on documentary sources such as autos and bandos of Good Government, ordinances and representations. These regulations were reproduced or produced locally by the cabildos. They inform about the local reality of the city council and the neighborhood considering the universal reality of the Crown. The article examines various social dimensions related to the bodies, and thereby reviews the use of the Thompson's and Scott's model and its application relating bodily practices and expressions with the regulations of colonial authorities of the 18th century. Thus, the notion of moral economy can be applied in a broader sense for analytical observations in relation to the human body.
\end{abstract}

Keywords: Moral economy; body; control; Jujuy; Ancien Regime. 


\section{Grit Kirstin Koeltzsch}

Die Entfernung aber des Guten, was wir in uns bewirken sollen, von dem Bösen, wovon wir ausgehen, ist unendlich und sofern, was die That, d.i. die Angemessenheit des Lebenswandels zur Heiligkeit des Gesetzes, betrifft, in keiner Zeit erreichbar.

Imanuel Kant ${ }^{2}$

\section{Introducción}

El antropólogo francés David Le Breton le asigna al cuerpo una posición simbólica a partir del imaginario social y las representaciones sociales que "hacen" al cuerpo. El mismo queda entonces como resultado de una construcción social y cultural. ${ }^{3}$ En este sentido podemos estudiar las representaciones históricas, socio-culturales y psicológicas del cuerpo donde las corporalidades contribuyen a la visión del mundo como construcción simbólica ${ }^{4}$, o sea, el cuerpo representando la construcción simbólica de una sociedad.

Por mucho tiempo el cuerpo no ha sido considerado como importante en los estudios culturales, entre otras cuestiones, porque se presuponía la división entre lo natural (el cuerpo biológico) y lo cultural ${ }^{5}$. Esto fue lo que daba lugar a que se estudie por separado el aparato biológico del hombre sin tener en cuenta los aspectos históricosculturales o viceversa. ${ }^{6}$ A esta problemática se suma la tradición dualista a partir del pensamiento cartesiano, donde se asigna hasta inclusive más importancia a la mente que al cuerpo. Sin embargo, en varias disciplinas y de a poco se inició la preocupación acerca de los cuerpos y su relación con la sociedad, ya que no podemos percibir el mundo ni

\footnotetext{
2 Imanuel Kant, Gesammelte Schriften, AA Band VI. Die Religion innerhalb der blossen Vernunft (Berlin: Preußische Akademie der Wissenschaften, 1793), 66. [Pero la distancia entre el bien que debemos conseguir en nosotros mismos y el mal del que partimos es infinita y, en lo que se refiere al hecho, es decir, la adecuación del modo de vida a la santidad de la ley, no se puede alcanzar en ningún momento. (traducción propia)].

${ }^{3}$ David Le Breton, Antropología del Cuerpo y Modernidad (Buenos Aires: Nueva Visión, 1995), 14.

${ }^{4}$ David Le Breton, Antropología del Cuerpo..., 13.

5 Roy Porter, "Historia del cuerpo", en Formas de hacer historia, ed. Peter Burke (Madrid: Alianza, 1993).

${ }^{6}$ Jacques Le Goff y Nicolas Truong, Una historia del cuerpo en la Edad Media (Barcelona: Paidós, 2005), 18.
} 
explicarlo sin el cuerpo, ${ }^{7}$ es decir, el concepto de cuerpo inevitablemente atraviesa las diferentes disciplinas.

En cuanto al comportamiento económico, desde las teorías clásicas se pensaba en términos de la racionalidad económica del hombre, basándose en las propuestas de la economía clásica de Smith y Ricardo, así como lo plantea John Stuart Mill en relación al homo xconomicus, y de que el hombre es un ser netamente racional motivado por el deseo de riqueza con un comportamiento enfocado en la maximización de utilidad. Resulta que la Economía Política considera a la humanidad como ocupada únicamente en la adquisición y consumo de riqueza. ${ }^{8}$ Por otro lado, antropólogos como Polanyi, Mauss y Sahlins ${ }^{9}$ han destacado que la conducta económica en sociedades tradicionales fue dirigida por criterios sociales, normas morales y proscripción ritual. Esto demuestran las actividades que se difieren considerablemente de las del homo œeconomicus, por ejemplo a través de patrones de reciprocidad, el intercambio de bienes o la economía moral del Antiguo Régimen. Encontramos en los documentos de los autos, bandos y otros reglamentos estatales para el Buen Gobierno de la ciudad de Jujuy en los siglos XVIII y primeras décadas del XIX este juego entre el orden y la moral, el consenso para la juntada de cuerpos en ciertas ocasiones y otras prácticas relacionadas a los cuerpos. De esta manera tal vez podamos entender las reflexiones de Kant como yuxtaposición de lo físico y lo moral, o como lo hace Norbert Götz, ${ }^{10}$ quien propone entender economía moral a partir del enunciado de Kant cuando habla de "la adecuación del modo de vida". ${ }^{11}$

Ahora bien, considerando estos aspectos económicos, históricos y culturales, queremos reafirmar la importancia del cuerpo como actor histórico, en la línea de

\footnotetext{
${ }^{7}$ Heidrun Zettelbauer, "Positionierungen”. En Verkörperungen. Transdisziplinäre Analysen zu Geschlecht und Körper in der Geschichte, eds. Heidrun Zettelbauer et.al. (Göttingen: V\&R unipress, 2017), 10.

8 John Stuart Mill, Essays on Some Unsettled Questions of Political Economy. Essay 5. Second Edition (London: Longmans, Green, Reader, And Dyer, 1874), 97.

9 Karl Polanyi et.al., Comercio y mercado en los imperios antiguos (Barcelona: Labor, 1974). Marcel Mauss, Sociología y Antropología (Madrid: Tecnos, 1979); Marshall Sahlins, Economía de la Edad de Piedra (Madrid: Akal, 1983).

${ }^{10}$ Norbert Götz. "Moral economy: its conceptual history and analytical prospects", Journal of Global Ethics 11, no.2 (2015): 147-162,

${ }^{11}$ Imanuel Kant, Gesammelte Schriften..., 66.
} 


\section{Grit Kirstin Koeltzsch}

pensamiento de Marc Bloch quien tempranamente señaló la historicidad del cuerpo entendiéndolo desde una perspectiva holística. Además, para quien "la verdadera historia se interesa en el hombre íntegro, con su cuerpo, su sensibilidad, su mentalidad y no solamente sus ideas y sus actos"12 y así podemos entender el hombre en su sociedad, en el tiempo y espacio.

Respecto al caso de estudio, Jujuy en el siglo XVIII es una ciudad ubicada en el Tucumán que sirve de eslabón a la región económica motorizada por la minería potosina y que relaciona el puerto de Buenos Aires con los mercados mineros meridionales bajo la autoridad del Virreinato del Perú, la Audiencia de Charcas, y finalmente del Río de la Plata. Por lo que aunque pequeña, es una vital estación de circulación dentro del "sistema de la economía regional" del Virreinato peruano y luego del rioplatense. ${ }^{13}$

En la primera parte del artículo se revisan algunos aspectos acerca del control y el disciplinamiento corporal en el espacio colonial de Jujuy en el siglo XVIII en relación a Sozialdisziplinierung (disciplinamiento social) en el Antiguo Régimen y sus particularidades. Luego, consideramos la posibilidad de ampliar la noción de economía moral en base de las teorías existentes. Se identificarán los aspectos corporales surgidos en el mercado (como espacio de relaciones de poder) de la economía moral. En la tercera parte se analizan los casos concretos estableciendo cinco categorías identificadas en los documentos. Se concluye con algunas reflexiones acerca de la relación al comportamiento social en el Antiguo Régimen y que estos documentos no solamente informan de la dominación, sino también de las diversas prácticas subalternas en este espacio y período histórico.

En la investigación partimos de la premisa que las masas populares no solamente actúan de manera irracional, compulsiva o motivada por el hambre, y no siempre se resuelven los conflictos con rebeliones, sino también con consensos. De esta manera el trabajo se entiende como una propuesta para fomentar a examinar diversas dimensiones

\footnotetext{
12 Jaques Le Goff, "Prefacio", en: Apología para la Historia o el oficio de historiador de Marc Bloch (México: Fondo de Cultura Económica, 2001), 15.

13 Carlos Sempat Assadourian, El sistema de la Economía Colonial. El mercado interior. Regiones y espacio económico (México: Editorial Nueva Imagen, 1983), 161.
} 
sociales relacionadas a los cuerpos, lo que permite revisar el uso del modelo de economía moral de Thompson y Scott y su aplicación, con el fin de relacionar prácticas y expresiones corporales con las reglamentaciones de las autoridades coloniales del siglo XVIII. Por lo tanto, la noción de economía moral puede ser utilizada en un sentido más amplio para observaciones analíticas en relación al cuerpo del ser humano. La fuente documental constituye una selección de autos, bandos y reglamentos estatales encontrados en archivos de Jujuy ${ }^{14}$ que efectivamente fueron aplicados para el Buen Gobierno de la ciudad de Jujuy en los siglos XVIII y primeras décadas del XIX.

\section{Control y disciplinamiento. Jujuy en el siglo XVIII}

Para acercarnos a la realidad en cuanto al control de los cuerpos, las reglas y las penas por no cumplirlas, en este estudio trabajamos con una muestra de documentos que describen las normas estatales para el Buen Gobierno de la ciudad de Jujuy en los siglos XVIII y primeras décadas del XIX. Los bandos y autos de buen gobierno fueron un mandamiento de autoridad competente dirigido a los habitantes de una ciudad indiana (en su triple jurisdicción espacial de casco urbano, ejido y dehesa), con un conjunto articulado de disposiciones sobre diversas materias relativas a la vida local que se daban a conocer públicamente. Eran reglamentaciones institucionalizadas del dominio hispano, que permiten conocer cómo se representa estatalmente la economía del poder. Considerados como un «derecho de aplicación popular», trataban distintos aspectos mundanos de la vida local y estaban dirigidos a todos con un formato de elaboración y difusión amplio. ${ }^{15}$ Por eso no extraña encontrar autos y bandos regulando la vida local en las regiones y

\footnotetext{
${ }^{14}$ Mayormente provienen del Archivo Histórico de Jujuy (AHJ), el Archivo Ricardo Rojas (ARR) y el Archivo de Tribunales de Jujuy (ATJ).

15 Víctor Tau Anzoátegui, Los bandos de buen gobierno del Río de la Plata, Tucumán y Cuyo (época hispánica) (Buenos Aires: IIHD, 2004), 17-22.
} 


\section{Grit Kirstin Koeltzsch}

jurisdicciones vecinas al Tucumán, como Asunción del Paraguay, Reino de Chile y Buenos Aires. ${ }^{16}$

Los rubros a los que atienden los autos y bandos de Jujuy y del Tucumán son similares a los de otros distritos como Asunción del Paraguay: administración de la justicia y buen gobierno o policía. Tratando materias como la religión y la moral pública; la población y seguridad social; el orden edilicio, el tránsito y ordenamiento de la vía pública, la limpieza y la salubridad urbana y rural; los abastos, pulperías, tiendas y carnicerías; los juegos, fiestas y bailes; y las relaciones socio laborales del trabajo, el vagabundaje y la pobreza; los regímenes de propiedad privada y la vida pública y particular de la familia, la casa y los vecinos; etc. ${ }^{17}$ Con el análisis de los documentos encontrados en Jujuy queremos visibilizar las dinámicas entre disciplina social y performance, ya que por un lado están presentes los mecanismos de control, pero también la acción social a través de diversas actividades como juntarse en las pulperías, jugar, danzar y tocar el tambor de lo que nos informan los autos y bandos. Por lo tanto, podemos decir que lograr disciplina social no es un acontecimiento recto, sino es un proceso histórico que requiere negociación, en el sentido del uso del concepto Gerhard Oestereich según el cual "Sozialdisziplinierung ist eine idealtypische Begriffsbildung, die historische Ereignisse des geistigen und materiellen Lebens, religiösethische Vorstellungen sowie die rechtliche und ökonomisch-soziale Wirklichkeit auf einen abstrakten Nenner bringt". ${ }^{18} \mathrm{O}$ sea, encontramos todos los aspectos mencionados, tanto económicos, jurídicos como sociales y espirituales como base de análisis dentro del marco de la economía moral.

\footnotetext{
${ }^{16}$ Víctor Tau Anzoátegui, Los bandos de...; Herib Caballero Campos, Los bandos de buen gobierno de la Provincia del Paraguay, 1778-1811 (Asunción del Paraguay: Arandura Editorial, 2007); Francis Goicovich, "La permeable frontera de la sociedad finicolonial: una aproximación al uso jurídico del imaginario social (Santiago de Chile, 1804)", Revista Escuela de Historia, no. 5 (2006): 3-24.

${ }^{17}$ Víctor Tau Anzoátegui, Los bandos de... Herib Caballero Campos, Los bandos de...

${ }^{18}$ La "Disciplina Social" es un concepto ideal que busca encontrar un denominador abstracto para los acontecimientos históricos de la vida espiritual y material, ideas ético-religiosas, así como para la realidad jurídica y económico-social (traducción propia). En Winfried Schulze, "Gerhard Oestreichs Begriff der Sozialdisziplinierung in der Frühen Neuzeit“, ZHF, no. 14 (1987): 265-302.
} 


\section{La economía moral del cuerpo en el Antiguo Régimen hispano-colonial}

Cabe destacar que el disciplinamiento como regulador en el Antiguo Régimen no solamente fue posible desde la imposición institucional, sino también requería el consentimiento de los receptores sociales. ${ }^{19}$ Teniendo en cuenta las diferentes prácticas, costumbres y rituales de una población heterogénea, el orden social no se podía instalar únicamente desde la autoridad estatal. Juegan en conjunto los diferentes lados, como bien reconoce Macarena Cordero, "el control social puede ser ejercido 'desde arriba' como también 'desde abajo', lo que crea a su vez la posibilidad de la interacción y relación entre diversos mecanismos de control", ${ }^{20}$

En cuanto al espacio al que refiere la muestra, se trata de Jujuy, una ciudad ubicada en el Tucumán bajo la administración primero de la gobernación del Tucumán y luego de la Intendencia de Salta del Tucumán. Se trata de una jurisdicción que en el siglo XVIII tiene una óptima estructura económica y social mercantil de Antiguo Régimen, porque sirvió como eslabón entre la minería potosina y el puerto de Buenos Aires. ${ }^{21}$ Además de configurar, al igual que las otras ciudades del Tucumán de San Miguel de Tucumán, Salta y Santiago del Estero, una frontera de guerra violenta en el Chaco de la que participan españoles, criollos e indígenas. ${ }^{22}$

\footnotetext{
${ }^{19}$ Macarena Cordero, "Precariedad del proyecto disciplinador de la coronan e iglesia en el siglo XVIII. Las doctrinas periféricas de la diócesis de Santiago de Chile", en Formas de control y disciplinamiento. Chile, América y Europa, siglos XVI-XIX, eds. Verónica Undurraga y Rafael Gaune (Santiago de Chile: Uqbar/Instituto Riva-Agüero, 2014), 143-166.

${ }^{20}$ Macarena Cordero, "Precariedad del proyecto...", 144.

${ }^{21}$ Zacarías Moutoukias, "Gobierno y sociedad en el Tucumán y el Río de la Plata, 1550-1800”, en Academia Nacional de la Historia, Nueva Historia Argentina, tomo 2 La sociedad colonial (Buenos Aires: Sudamericana, 2000), 355-411; Luisa Consuelo Soler Lizarazo y Enrique N. Cruz, "Un proyecto innovador en una hacienda de añil del Tucumán (fines del siglo XVIII)", Boletín Americanista, no. 72-1 (2016): 155177.

22 Juan Carlos Garavaglia, "La guerra en el Tucumán colonial: sociedad y economía en un área de frontera (1660-1760)", HISLA, IV (1984): 21-34; B. Vitar, Guerra y misiones en la frontera chaqueña del Tucumán (1700-1767) (Madrid: Consejo Superior de Investigaciones Científicas, 1997); S. E. Mata de López, "Las fronteras coloniales como espacios de interacción social. Salta de Tucumán (Argentina), entre la Colonia y la Independencia", Dimensión Antropológica, no. 33 (2005): 69-90; Enrique N. Cruz, "La nueva sociedad de frontera. Los grupos sociales en la frontera de San Ignacio de Ledesma, Chaco occidental, finales del siglo XVIII", Anuario de Estudios Americanos 58, no.1 (2001): 135-160; D.J. Santamaría, Chaco Gualamba. Del monte salvaje al desierto ilustrado (Jujuy: Cuadernos del Duende, 2007).
} 


\section{Grit Kirstin Koeltzsch}

De esta manera, y como parte del "espacio peruano", Jujuy será una pequeña estación útil dentro del "sistema de la economía regional", ${ }^{23}$ porque sirve con sus pasturas de alfalfares para el engorde de ganado de mulas en travesía a los mercados mineros andinos y en el nodo de distintos circuitos mercantiles que vinculaban los puertos del Callao con el de Buenos Aires y el Reino de Chile y la región de Cuyo con Potosí y viceversa. ${ }^{24}$ Revisaremos a continuación la relación entre los cuerpos y cómo podemos vincular el disciplinamiento social en el marco de la economía moral en Jujuy del siglo XVIII.

\section{Cuerpos en el espacio colonial. Noción ampliada de economía moral}

El concepto de economía moral fue desarrollado por el historiador Thompson, ${ }^{25}$ sin embargo, la noción fue adoptada y ha sido utilizada con más frecuencia por académicos de otras disciplinas, como la sociología, la antropología y las ciencias políticas. ${ }^{26}$ Thompson ofrece un modelo de protesta, de cultura política y que incluyen las relaciones entre las élites y los dominados en el ambiente de la clase trabajadora en áreas urbanas en el siglo XIX desde la mirada marxista. Por otro lado, está el aporte de Scott que se concentra en los productores, el campesinado en las sociedades colonizadas o anteriormente colonizadas. Como consecuencia, sus reflexiones giran en torno a la subsistencia campesina de las economías sobre todo en países asiáticos. Así para el

\footnotetext{
${ }^{23}$ Carlos Sempat Assadourian, El sistema de la ..., 161.

24 Carlos Assadourian y S. Palomeque, "Los circuitos mercantiles del "interior argentino" y sus transformaciones durante la Guerra de la Independencia (1810-1825)", en La historia económica y los procesos de independencia en la América hispana, comp. S. Bandieri (Buenos Aires: Prometeo Libros, 2010), 49-70. Pablo Lacoste et.al., "Cobre labrado, alambique y aguardiente. Chile y Argentina, 15861850", Quinto Sol, no. 20-1 (2016): 1-29. Luisa Consuelo Soler Lizarazo, "Redes de comercialización de la yerba mate a partir de las operaciones mercantiles de Salvador Trucíos, Chile (1758-1798)", RIVAR, no. 3-9 (2016): 26-49.

25 Edward P Thompson, "The Moral Economy of the English Crowd in the Eighteenth Century", Past \& Present, no. 50 (1971): 76-136.

${ }^{26}$ Peter Burke, History and Social Theory (Ithaca: Cornell University Press, 1993), 1-2.
} 
historiador James $\mathrm{Scott}^{27}$ el concepto de economía moral permite una mejor comprensión de las raíces normativas de la política campesina: cómo las transformaciones económicas y políticas de las colonias han desafiado las concepciones de equidad social de los campesinos. En esta línea, con el ejemplo de un espacio hispanoamericano, él de las haciendas en México, Eric Wolf ${ }^{28}$ describe cómo las transformaciones económicas y políticas de la época colonial han desafiado las concepciones de los campesinos sobre la equidad social. Así también explica acerca de los compromisos, ya que las comunidades indígenas en las áreas rurales no solamente han mantenido sus concepciones, sino también sus lenguas y sus patrones culturales que no son para nada homogéneos. Lo que dice Wolf al respecto es lo siguiente:

Yet this meant, too, that the haciendas were forced to share the landscape with communities of Indians. Under Spanish rule, these had received the special protection of the state. They had been granted the legal status of corporations, and each community was allowed to retain a stipulated amount of land under its own communal management, as well as its own autonomous communal administration. ${ }^{29}$

Como hemos mencionado anteriormente, en este trabajo queremos hacer hincapié en un uso de economía moral como noción ampliada, entendida como un enlace vertical en ambas direcciones, o sea, como concepción dialéctica en la cual el poder se conjuga tanto top-down como bottom-up. Además, entender economía moral como un conjunto de valores en cierta relación con la preocupación por la subsistencia anclada en la reciprocidad, así en acuerdo con el planteo de Siméant:

Moral economy can be understood as a set of values derived from living conditions that are marked by a preoccupation with subsistence, linked to the reciprocal expectations of elites and the people which are at once pragmatic and

\footnotetext{
27 James Scott, The Moral Economy of the Peasant. Rebellion and Subsistence in Southeast Asia (London: Yale University Press, 1976).

${ }^{28}$ Eric Wolf, Peasant Wars of the Twentieth Century (New York: Harper and Row, 1969).

${ }^{29}$ Eric Wolf, Peasant Wars of...., 4.
} 


\section{Grit Kirstin Koeltzsch}

normative, and which concern the fair distribution of wealth and the responsibility of leaders. ${ }^{30}$

Así pues, lo que tratan de proponer Thompson y Scott es pensar en otros esquemas, es decir, enfrentarse a las concepciones mecánicas acerca de las masas populares, sugiriendo pensar en un vínculo entre las expectativas populares y la influencia de las autoridades ${ }^{31}$.

Ahora bien, lo que se ha elaborado es una muestra a partir de la cual se puede deducir distintos aspectos que refieren a lo corporal: como el control de consumo de bebidas y las penas corporales; el control del juego y sus penas corporales; el control de la movilidad corporal espacial y laboral de los trabajadores, prohibiéndose el "vagabundeo" o sea la libertad para moverse, trabajar y contratarse con patrones; la prohibición de "portación" de armas con los castigos corporales; la sujeción y quietud de las personas establecidas a partir del matrimonio; la prohibición de las reuniones, diversiones y bailes en general y de negros en particular; los castigos corporales por venta de alhajas; y el cantar y vestir disfraces públicamente; etc. Estas y otras características pueden verse en todas estas reglamentaciones, y de esta manera nos informan de la omnipresencia de los aspectos corporales en las normas que refieren a lo empírico y cotidiano de la realidad social.

Teniendo en cuenta las características de la documentación utilizada y el período histórico, por varios motivos se puede interpretar que este tipo de documentos permiten conocer cómo se establecía la economía moral del cuerpo. En primer lugar, porque el cuerpo constituye el locus de las biopolíticas, segundo, porque las relaciones entre dominadores y subalternos resultaron del juego entre imposición y consentimiento, y tercero, porque la mecánica de publicidad de esta reglamentación tiene un evidente carácter popular. Constituyéndose con estos tres elementos del mercado de la economía moral, permitiéndonos distinguir la performance de lectura en bando en la plaza de la

\footnotetext{
${ }^{30}$ Johanna Siméant, "Three bodies of moral economy: the diffusion of a concept", Journal of Global Ethics 11, no.2 (2015): 163-175.

${ }^{31}$ Johanna Siméant, "Three bodies of moral economy: the diffusion of a concept", 171.
} 
ciudad, distintos aspectos biopolíticos y las relaciones entre el poder y lo popular del cuerpo en este período y espacio histórico.

A continuación, revisamos los ejemplos concretos en cada una de estas reglamentaciones estatales y se identificarán los aspectos corporales surgidos en el mercado (como espacio de relaciones de poder) de la economía moral.

\section{Reglamentaciones estatales con relación al control corporal}

Cabe destacar que la frecuente mención y omnipresencia de asuntos con relación al cuerpo es en sí un indicio de la importancia de las expresiones corporales. Para el análisis fue necesario dividir los diferentes aspectos relacionados al cuerpo en grupos según la información encontrada en las fuentes documentales. Por lo tanto, en principio se han identificado cinco categorías que refieren directamente al cuerpo y que articulan la prohibición, penas y el control corporal, además que hacen referencia a prácticas corporales efectivas. Los denominamos de la siguiente manera:

1) Borracheras, juegos y juntas: los "cuerpos juntados". Se trata de una práctica constituida por la embriaguez y el juego, de manera general constituido como espacio de la "reunión". Esto constituye una situación especial, ya que la conducta es problemática, porque la embriaguez y el juego crean un espacio en el que los cuerpos se juntan, sin tener en cuenta las diferencias étnicas, clasistas y de dominación.

2) Vagamundos y conchabo: cuerpos vagamundos y trabajo. En este caso, se ha identificado que la preocupación de las autoridades es evitar el vagabundeo y controlar laboralmente el cuerpo de las personas. En este contexto, las autoridades siempre van a relacionar la libertad de movilidad del cuerpo con la necesidad de fijación del cuerpo: vagabundeo y trabajo.

3) El delito del amancebamiento: cuerpos casados y amancebados. En relación a lo anteriormente mencionado acerca del control y la fijación del cuerpo, se considera 


\section{Grit Kirstin Koeltzsch}

que el matrimonio significa fijar, retener y ayudar a controlar los cuerpos de las personas. De allí proviene la orden de que los casados vivan con sus esposas y a la vez condenar el amancebamiento, porque este mismo no fija el cuerpo del esposo a un solo lugar.

4) Cabalgaduras y tránsito: cuerpos encabalgados. Se entiende que el cuerpo es poderoso, y es aún más poderoso cuando aumenta su envergadura. De allí que se trata de controlar el tránsito y la ocupación del espacio que los cuerpos de las personas realizan cuando cabalgan, ya que significa aumentar su envergadura.

5) La performance bando: cuerpos sin color. Con esta categoría nos referimos a la teatralización a través de la cual se proclaman las normas por los pregoneros en las plazas públicas. Esta performance mayormente es acompañada por el toque de tambores y cajas. En cuanto a los "cuerpos sin color", esto porque las normas se aplican a todas las personas, y así también los pregoneros suelen ser en su mayoría "negros ladinos".

De manera general, recorriendo los autos y bandos podemos detectar cómo se expresa esta dialéctica bottom-up/top-down en las normas, aparecen menciones de demandas y quejas para que el buen gobierno, que se reconocen también desde las autoridades. Un ejemplo es el desabastecimiento de carne cuando no se cumple con la obligación de proveer suficiente al mercado y se mencionan que "los vecinos como los forasteros, quienes con mucha razón gravemente se quejan del poco prolijo gobierno". ${ }^{32}$ Esta información de alguna manera indica la legitimidad de la negociación en la economía moral.

En todos los documentos analizados, la primera categoría tratando el tema de las borracheras y el control de venta de alcohol es la que más aparece, o sea, aparentemente el control sobre los cuerpos juntados fuera del horario de trabajo parece ser de suma importancia, sin embargo, no está prohibida. Considerando que en los trabajos de cosecha

\footnotetext{
32 "Representación solicitando Bando del procurador general de la ciudad de San Salvador de Jujuy, Bernardo Espinosa al cabildo justicia y regimiento de Jujuy, 7 de febrero de 1757”.
} 
o de otro tipo, los cuerpos tenían que estar juntos, así también se juntaban en el tiempo libre. Algunos documentos en particular dejan entender que había este espacio de negociación de las normas cuando se señala una prohibición, pero no de manera completa. Un ejemplo es la mención a la cantidad de alcohol que se permite tomar en ciertas ocasiones y que resulta ambigua. Es decir, cuando se estipula vender "ni mucha, ni poca" cantidad de vino como en el caso aquí citado: “(...)durante la celebración de los divinos oficios, y hasta que se concluya la misa parroquial, no se venda por los pulperos aguardiente, ni vino en mucha, ni en poca cantidad a ninguna clase de personas(...)" ${ }^{33}$ Se podría interpretar que es posible tomar/vender muy poca cantidad de alcohol, ya que no se menciona ninguna prohibición total. Así también se negocian los espacios de juntarse sin importar categorías como raza, género o clase, ya que la mayoría de las menciones inician con la frase "que ninguna persona sea del sexo y naturaleza que fuere(...)". En cuanto a los juegos se clasifica por ejemplo en "juegos extraordinarios", o sea, no están totalmente prohibidos. En cuanto a la aplicación de penas, sí se distingue entre nobles y plebe.

Ítem, que ninguna persona de cualesquier calidad y condición que sean no permitan en sus casas tiendas ni pulperías Juegos extraordinarios como de Naipes Dados y suertes, ni menos la gente plebe y se recoja en los montes ranchos, ni barrancas a usar de sus juegos, so pena que a los primeros se les arrancaran 25 pesos de multa para gastos de justicia, y a los jugadores siendo de la Noble, además de volver lo ganado, y a los segundos por la primera 50 azotes y un mes de cárcel, y por la segunda cien azotes y un año de presidio a ración y sin sueldo=

Por otra parte, en cuanto al baile y la música, lo que sigue causando un problema para las autoridades son los así llamados "bailes de negros” que se prohíben. "Que los negros no se junten a los escandalosos bailes que acostumbran con su tambor bajo la pena

\footnotetext{
33 “Auto de la intendencia de Salta del Tucumán, Salta, 10 de abril de 1794. AHJ, ARR, Caja XLII, Legajo 1, Documento de abril 1794, 8 fojas (11-14)".

34 "Auto de Buen Gobierno del teniente de gobernador y alcaldes de Jujuy, 10 de enero de 1781". ATJ, carpeta 54, legajo 1765 ”.
} 


\section{Grit Kirstin Koeltzsch}

de cincuenta azotes siempre, que tal hicieren=". 35 Así pues siguen siendo considerados vulgares y peligrosos, ya que la fuerza del tambor y los movimientos chocan con el cuerpo y la mente del español. Mientras los bailes religiosos de los indios fueron tomados como "evidencia consoladora de la conversión del nativo", 36 aunque no entendieron tampoco los mensajes sutiles y el mimetismo del indígena, por parte de las autoridades, en primer plano lo consideraron como exitosa conversión al cristianismo.

Ahora bien, muy relacionado al tema del control de los cuerpos es la categoría del vagamundo y conchabo, ya que a partir del trabajo se deja controlar mejor los cuerpos, y así se apunta a evitar vagancia, ociosidad y mal entretenimiento, ya que "huyendo del trabajo distraen a los que viven ocupados". ${ }^{37}$ Esta categoría identificada en los documentos aparece muy frecuentemente, lo que podemos atribuir a la vinculación de la borrachera con vagancia, y esto llevaría a la distracción del trabajo. Hay autos que indirectamente prohíben la venta de bebidas en respuesta a la medida de la iglesia con el fin de evitar las juntas de la gente que trabaja, y esto apunta a evitar la vagancia. ${ }^{38}$

En cuanto al delito del amancebamiento que se menciona solamente en algunos documentos, ahí se ordena averiguar e identificar a aquellos que son casados y si residan con sus esposas o no. Por lo tanto, llaman a los casados que estén con sus esposas haciendo vida maridable.

(...)se precise a todos los casados vayan a hacer vida maridable con sus mujeres; para que tenga su más puntual y debido cumplimiento, lo determinado por su alteza, debían de mandar y mandaron que todos los casados que se hallasen en

\footnotetext{
35 “Auto y bando del gobernador del Tucumán Antonio de Arriaga, Salta, 24 de julio 1774. Publicado en bando en Jujuy, 6 de enero de 1777 por los alcaldes de Jujuy”. AHJ, ARR, C. IX, legajo 2, legajillo 2, fojas 131-134.

36 Deborah A Poole, “Accommodation and Resistance in Andean Ritual Dance", The Drama Review 34, no. 2 (1990): 98-126.

37 “Auto y bando de los alcaldes ordinarios de primer y segundo voto de la ciudad de Jujuy, Jujuy, 18 y 19 de enero de 1794". AGN, Tribunales, IX, 38-4-5, exp. 24, fs. 3-4 v. Citado por Víctor Tau Anzoátegui, Los bandos de...

38 "Auto de los alcaldes de primer y segundo voto de la ciudad de Jujuy, Jujuy, 10 de octubre de 1795 y oficio de los curas rectores de la matriz de Jujuy, Jujuy, 8 de octubre de 1795”. AHJ, ARR, Caja XLII, legajo 1 , documento de octubre 1795 , fojas 30 y 31 .
} 


\section{La economía moral del cuerpo en el Antiguo Régimen hispano-colonial}

esta ciudad, y en el distrito de esta jurisdicción salgan a hacer vida con sus mujeres en el término de quince días(...). ${ }^{39}$

Aquí se trata de controlar la sexualidad de los cuerpos casados y amancebados, garantizar la reproducción y de alguna manera ir regulando cierta conducta en este sentido, cuando en realidad nadie puede controlar absolutamente los dormitorios de las casas o actividades sexuales en otros lugares. En los bandos no se mencionan exactamente cuál sería el comprobante del no cumplimiento y tampoco se expresan claramente las penas para este "delito". Queda en negociación de los actores sociales de denunciar o no, o cumplir o no esta regla.

En relación con las cabalgaduras y tránsito detectamos que en algunas instancias se prohíbe tener la cabalgadura en la puerta del cementerio durante la misa o estacionar el caballo en calles y esquinas, por ejemplo:

Que ninguna persona sea del sexo que fuese los días de fiesta o de trabajo no tengan sus cabalgaduras en las puertas de los cementerios sino que los amarren en el rollo o en otra parte distante de los lugares sagrados porque es cierto que con este hecho se origina el poco respeto y veneración del culto de $\operatorname{Divino}(. . .)^{40}$

Se entiende que los cuerpos encabalgados se encuentran en una situación especial, ya que constituyen un espacio de negociación, porque el cuerpo a caballo es poderoso junto al cuerpo de un animal fuerte como es el caballo. Puede ser la razón por la cual se prohíbe galopear en la calle, a toda persona que "corra, ni galopee a caballo por las calles de día ni de noche bajo la pena de perder el caballo y jaez"41. Cabe destacar que de alguna

\footnotetext{
39 “Auto de la Real Audiencia de Charcas sobre vida maridable de las personas de la frontera, La Plata, 14 de enero de 1771. Toma de razón y repetición del gobernador del Tucumán Gregorio de Matorras, Salta, 26 de septiembre de 1774. Publica en Bando en la ciudad de Jujuy y distintos parajes de la frontera, alcaldes del cabildo de Jujuy, Jujuy, Río Negro, Jujuy, 7, 16 y 19 de octubre de 1774”. AHJ-ARR, Caja IX (9), Legajo 2, Documento del año 1774, 11 fojas.

40 “Auto y bando de los alcaldes ordinarios de primer y segundo voto de la ciudad de Jujuy, Jujuy, 7 de febrero de 1778, en bando el 8 de febrero de 1778". ATJ, Carpeta 52, legajo 1723.

41 “Auto y bando del gobernador del Tucumán Antonio de Arriaga, Salta, 24 de julio 1774. Publicado en bando en Jujuy, 6 de enero de 1777 por los alcaldes de Jujuy”. AHJ, ARR, C. IX, legajo 2, legajillo 2, fojas 131-134.
} 


\section{Grit Kirstin Koeltzsch}

manera las autoridades reconocen la fuerza que tiene la presencia del cuerpo, tanto del animal o como él del ser humano, y disimulan su miedo bajo el pretexto de un "pueblo civilizado" no se relaciona con "bestias", por lo tanto, se prohíbe "la introducción de potros, mulas y otros animales feroces a las calles de la ciudad con el pretexto de domesticarlos" ${ }^{42}$ con la pena de "perder la bestia". Así pues, las penas no eran más que perder el animal o pagar el daño en el caso de causar alguno al galopear en la calle, pero indica un cierto margen y consenso para esta expresión corporal.

Como última categoría, lo que aquí llamamos "la performance bando", se refiere al acto performativo de leer el bando en una plaza pública frecuentada por la gente y el toque de caja junto a la voz del pregonero. A veces se realizaba este acto en días feriados religiosos o el día domingo para su mayor alcance en la población, "por ser el de más concurso a toque de caja a usanza de guerra en la plaza pública y esquinas de las calles acostumbradas. ${ }^{43}$ En esta situación se reconoce la fuerza del toque de caja lo que se trata de evitar en los bailes de negros. Por otra parte, en relación a los “cuerpos sin color", porque se estipulan las normas para todos los cuerpos, independientemente del sexo o raza. Una cuestión que llama la atención que los pregoneros a menudo son mencionados como "negros ladinos" con oficio de pregonero, o sea, se reconoce esta capacidad de una persona negra hasta inclusive que sepan leer, ya que los documentos públicamente son leídos en voz alta. Por ejemplo, "En diecinueve de enero de corriente año, se publicó este auto por voz de Mariano Mármol, negro ladino que hacía de oficio de pregonero..."44 De alguna manera esto demuestra la participación y negociación desde "abajo" para "arriba",

\footnotetext{
42 "Auto y bando de los alcaldes ordinarios de primer y segundo voto de la ciudad de Jujuy, Jujuy, 18 y 19 de enero de 1794". AGN, Tribunales, IX, 38-4-5, exp. 24, fs. 3-4 v. Citado por Tau Anzoátegui, Victor, Los bandos de buen gobierno del Río de la Plata, Tucumán y Cuyo (época hispánica), Buenos Aires, IIHD, 2004, pp. 436-439.

43 Bando del cabildo de Jujuy, San Salvador de Jujuy, 8 y 10 de enero de 1768". AHJ, Caja XLII, Legajo 2, Documento del año 1768, 4 folios. El bando en foja 1 y 2.

44 "Auto y bando de los alcaldes ordinarios de primer y segundo voto de la ciudad de Jujuy, Jujuy, 18 y 19 de enero de 1794". AGN, Tribunales, IX, 38-4-5, exp. 24, fs. 3-4 v. Citado por Tau Anzoátegui, Victor, Los bandos de buen gobierno del Río de la Plata, Tucumán y Cuyo (época hispánica), Buenos Aires, IIHD, 2004, pp. 436-439.
} 
ya que el "negro ladino" que leía los reglamentos simbólicamente representaba el pueblo común, reforzado por su presencia del ser negro o mulato.

Finalmente, cabe aclarar que aquí encontramos documentos con la reglamentación escrita y comunicada públicamente al pueblo. No tenemos información en cuanto a la eficacia del control y en qué frecuencia sucedieron desviaciones de estas normas para establecer el límite del control. Lo que sí se informa sobre algunas estrategias de control, por ejemplo, en situación de fiestas donde más probable se encuentre a personas con actitud de vagabundeo, así llaman a la observación, ya que "con el concurso el día de fiesta en esta ciudad se puede ir averiguando cuales son los vagantes". ${ }^{45} \mathrm{Sin}$ embargo, para ver si estas medidas fueron eficientes, habrá de encontrar fuentes documentales que comenten acerca de la desobediencia corporal. En otros espacios como en Buenos Aires del siglo XVIII también se mencionan frecuentemente las "reuniones indecentes" realizadas por negros de ambos sexos. ${ }^{46}$ Las prácticas sí existían y fue no tan fácil de prohibirlas. Relacionándolo con el tema de la prohibición de los "bailes de negros", observando los movimientos del presente podemos ver que han perdurado en las danzas latinas hasta el presente, y esto evidencia la "desobediencia" y puede marcar un límite del control. Como bien dice Bernand:

Los fandangos, danzones, tangos, sones, surgidos de la música lúbrica de las castas, que con tozudez pero con ineficacia los bandos reiterados de los virreyes se esforzaron en combatir año tras año, invaden inexorablemente todos los estratos de la sociedad, introduciendo lo sensual en la vida cotidiana. Sin lugar a dudas, el cuerpo en movimiento es la revancha del cuerpo mestizo y la característica más notable, hasta el día de hoy, de lo "latino". ${ }^{47}$

\footnotetext{
45 "Representación solicitando Bando del procurador general de la ciudad de San Salvador de Jujuy, Bernardo Espinosa al cabildo justicia y regimiento de Jujuy, 7 de febrero de 1757”.

${ }^{46}$ Ricardo Rodríguez Molas, La música y la danza de los negros en el Buenos Aires de los siglos XVIII y XIX (Buenos Aires: CLIO, 1957).

47 Carmen Bernand, "Los nuevos cuerpos mestizos de la América Colonial”, en Retóricas del cuerpo amerindio, eds. M. Gutiérrez Estévez y P. Pitarch (Madrid: Iberoamericana, 2010), 114.
} 


\section{Grit Kirstin Koeltzsch}

\section{Conclusión}

Con este acercamiento, aunque de manera breve, tratamos hacer una lectura acerca del control corporal en Jujuy del siglo XVIII a partir de las fuentes documentales y en el marco del concepto de la economía moral. Todas estas reglamentaciones aquí analizadas eran legítimas, en el sentido de que tenían el consenso de la población, porque teatralmente -en una performance de Antiguo Régimen-, para denominarlo de algún modo, además de que convocaba a los cuerpos de todos y constituía el espacio como espacio público.

La convocatoria de los cuerpos de todos, sin distinciones raciales, puede verse en que los que publicaban las reglamentaciones eran diversos: las mismas autoridades, pregoneros sin identificar, y pregoneros identificados como negros o mulatos. En cuanto al espacio constituido como espacio público, era porque se realizaba con sonido de cajas, que llenaba todo el espacio, luego porque se hacía en lugares de concurrencia de las personas y con su concurrencia: plazas y días de fiesta.

Para concluir, ante este panorama, hemos identificado los diversos aspectos corporales presentes en todos los autos y bandos de una u otra forma. Así también notamos que estos mismos no solamente informan de la dominación, sino también de las diversas prácticas subalternas y de una performance que hacía a la economía moral del Antiguo Régimen en este espacio y período histórico, ya que para que aparezcan estos aspectos en tantos reglamentos, primero tiene que estar presente la acción en la vida festiva, espiritual y ritual para luego buscar y lograr una Sozialdisziplinierung.

\section{REFERENCIAS}

Assadourian, Carlos Sempat. El sistema de la Economía Colonial. El mercado interior. Regiones y espacio económico. México: Editorial Nueva Imagen, 1983.

Assadourian, C. S. y S. Palomeque. "Los circuitos mercantiles del "interior argentino” y sus transformaciones durante la Guerra de la Independencia (1810-1825)". En La 
historia económica y los procesos de independencia en la América hispana, compilado por S. Bandieri, 49-70. Buenos Aires: Prometeo Libros, 2010.

Bernand, Carmen. "Los nuevos cuerpos mestizos de la América Colonial”. En Retóricas del cuerpo amerindio, editado por M. Gutiérrez Estévez y P. Pitarch, 87-116. Madrid: Iberoamericana, 2010.

Burke, Peter. History and Social Theory. Ithaca: Cornell University Press, 1993.

Caballero Campos, Herib. Los bandos de buen gobierno de la Provincia del Paraguay, 1778-1811. Asunción del Paraguay: Arandura Editorial, 2007.

Cordero, Macarena. "Precariedad del proyecto disciplinador de la coronan e iglesia en el siglo XVIII. Las doctrinas periféricas de la diócesis de Santiago de Chile”. En Formas de control y disciplinamiento. Chile, América y Europa, siglos XVI-XIX, editado por Verónica Undurraga y Rafael Gaune, 143-166. Santiago de Chile: Uqbar/Instituto Riva-Agüero, 2014.

Cruz, Enrique N. "La nueva sociedad de frontera. Los grupos sociales en la frontera de San Ignacio de Ledesma, Chaco occidental, finales del siglo XVIII". Anuario de Estudios Americanos 58, no. 1 (2001): 135-160.

Garavaglia, Juan Carlos. "La guerra en el Tucumán colonial: sociedad y economía en un área de frontera (1660-1760)”. HISLA, no. 4 (1984): 21-34.

Götz, Norbert. "Moral economy: its conceptual history and analytical prospects". Journal of Global Ethics, no. 11:2 (2015): 147-162. DOI: 10.1080/17449626.2015.1054556.

Goicovich, Francis. "La permeable frontera de la sociedad finicolonial: una aproximación al uso jurídico del imaginario social (Santiago de Chile, 1804)”. Revista Escuela de Historia, no. 5 (2006): 3-24.

Kant, Immanuel. Gesammelte Schriften, AA Band VI: Die Religion innerhalb der bloßen Vernunft. Sie Metaphysik der Sitten. Berlin: Preußische Akademie der Wissenschaften, 1793. Disponible en: https://korpora.zim.uni-duisburgessen.de/kant/aa06/Inhalt6.html. 


\section{Grit Kirstin Koeltzsch}

Lacoste, Pablo et.al. "Cobre labrado, alambique y aguardiente. Chile y Argentina, 15861850”. Quinto Sol 20, no.1 (2016): 1-29.

Le Breton, David. Antropología del Cuerpo y Modernidad. Buenos Aires: Nueva Visión, 1995.

Le Goff, Jaques. "Prefacio". En Apología para la Historia o el oficio de historiador, Marc Bloch, 9-33. México: Fondo de Cultura Económica, 2001.

Le Goff, Jacques y Nicolas Truong. Una historia del cuerpo en la Edad Media. Barcelona: Paidós, 2005.

Mata de López, S. E. "Las fronteras coloniales como espacios de interacción social. Salta de Tucumán (Argentina), entre la Colonia y la Independencia”. Dimensión Antropológica, no. 33 (2005): 69-90.

Mauss, Marcel. Sociología y Antropología. Madrid: Tecnos, 1979.

Mill, John Stuart. Essays on Some Unsettled Questions of Political Economy. Essay 5. Second Edition, London: Longmans, Green, Reader, And Dyer, 1874.

Moutoukias, Z. “Gobierno y sociedad en el Tucumán y el Río de la Plata, 1550-1800”. En Academia Nacional de la Historia, Nueva Historia Argentina. Tomo 2, La sociedad colonial, 355-411. Buenos Aires: Sudamericana, 2000.

Polanyi, Karl et.al. Comercio y mercado en los imperios antiguos. Barcelona: Labor, 1974.

Poole, Deborah A. "Accommodation and Resistance in Andean Ritual Dance". The Drama Review 34, no 2 (1990): 98-126.

Porter, Roy. "Historia del cuerpo". En Formas de hacer historia, editado por Peter Burke, 255-286. Madrid: Alianza, 1993.

Rodríguez Molas, Ricardo. La música y la danza de los negros en el Buenos Aires de los siglos XVIII y XIX. Buenos Aires: CLIO, 1957.

Sahlins, Marshall. Economía de la Edad de Piedra. Madrid: Akal, 1983. 
Santamaría, D. J. Chaco Gualamba. Del monte salvaje al desierto ilustrado. S.S. de Jujuy: Cuadernos del Duende, 2007.

Schulze, Winfried. "Gerhard Oestreichs Begriff der Sozialdisziplinierung in der Frühen Neuzeit“، $Z H F$, no. 14 (1987): 265-302.

Scott, James. The Moral Economy of the Peasant. Rebellion and Subsistence in Southeast Asia. London: Yale University Press, 1976.

Scott, James. Weapons of the Weak: Everyday Forms of Peasant Resistance. Yale: Yale University Press, 1985.

Siméant, Johanna. "Three bodies of moral economy: the diffusion of a concept". Journal of Global Ethics 11, no. 2 (2015): 163-175. DOI: 10.1080/17449626.2015.1054559.

Soler Lizarazo, L. C. "Redes de comercialización de la yerba mate a partir de las operaciones mercantiles de Salvador Trucíos, Chile (1758-1798)”. RIVAR 3, no. 9 (2016): 26-49.

Soler Lizarazo, L. C. y E. N. Cruz. "Un proyecto innovador en una hacienda de añil del Tucumán (fines del siglo XVIII)”. Boletín Americanista, no. 72-1 (2016): 155-177.

Tau Anzoátegui, Víctor. Los bandos de buen gobierno del Río de la Plata, Tucumán y Cuyo (época hispánica). Buenos Aires: IIHD, 2004.

Thompson, Edward P. "The Moral Economy of the English Crowd in the Eighteenth Century”. Past \& Present, no. 50, (1971): 76-136.

Vitar, B. Guerra y misiones en la frontera chaqueña del Tucumán (1700-1767). Madrid: Consejo Superior de Investigaciones Científicas, 1997.

Wolf, Eric. Peasant Wars of the Twentieth Century. New York: Harper and Row, 1969.

Zettelbauer, Heidrun. "Positionierungen”. En Verkörperungen. Transdisziplinäre Analysen zu Geschlecht und Körper in der Geschichte, editado por Heidrun Zettelbauer et.al, 9-44. Göttingen: V\&R unipress, 2017. 
Grit Kirstin Koeltzsch

Abreviaturas

AHJ, ARR Archivo Histórico de Jujuy, Archivo Ricardo Rojas

ATJ Archivo de Tribunales de Jujuy

AGN Archivo General de la Nación Argentina 